\title{
Icariin protects rats against 5/6 nephrectomy-induced chronic kidney failure by increasing the number of renal stem cells

Zhongdi Huang ${ }^{1}$, Liqun He $\mathrm{e}^{2,3,4}$, Di Huang ${ }^{2,3,4}$, Shi Lei ${ }^{5^{*}}$ and Jiandong Gao 2,3,4*

\begin{abstract}
Background: Chronic kidney disease poses a serious health problem worldwide with increasing prevalence and lack of effective treatment. This study aimed to investigate the mechanism of icariin in alleviating chronic renal failure induced by $5 / 6$ nephrectomy in rats.

Methods: The chronic renal failure model was established by a two-phased 5/6 nephrectomy procedure. The model rats were given daily doses of water or icariin for 8 weeks. The kidney morphology was checked by HE staining. The levels of blood urea nitrogen, serum creatinine, and serum uric acid were measured by colometric methods. The expression of specified genes was analyzed by quantitative real-time PCR and immunohistochemical staining. The number of renal stem/progenitor cells was analyzed by CD133 and CD24 immunohistochemical staining.
\end{abstract}

Results: Icariin protected against CDK-caused damages to kidney histology and improved renal function, significantly reduced levels of BUN, creatinine, and uric acid. Icariin inhibited the expression level of TGF- $\beta 1$ whereas upregulated HGF, BMP-7, WT-1, and Pax2 expression. Moreover, ccariin significantly increased the expression of CD24, CD133, Osr1, and Nanog in remnant kidney and the numbers of CD133 ${ }^{+} / \mathrm{CD} 24^{+}$renal stem/progenitor cells.

Conclusions: These data demonstrated that icariin effectively alleviated 5/6 nephrectomy induced chronic renal failure through increasing renal stem/progenitor cells.

Keywords: Chronic kidney failure, Icariin, Renal function, Renal stem cells

\section{Background}

The kidneys function as the cleansing and recycling apparatus of the body removing waste products of metabolism and water soluble wastes from blood, excreting urea and ammonium, reabsorbing water, glucose, and amino acids, by which they serve homeostatic functions such as the regulation of electrolytes, maintenance of acid-base and salt-water balances, and regulation of blood pressure. Besides, the kidneys also serve as an endocrine organ producing hormones calcitriol and erythropoietin among others, and the enzyme rennin. Chronic kidney disease (CKD) is a

\footnotetext{
* Correspondence: leis428@yahoo.com; gaojiandong@hotmail.com ${ }^{5}$ Collage of Biology and pharmacy, China Three Gorges University, 8 Daxue Road, Yichang, Hubei 443002, China

${ }^{2}$ Department of nephrology, Shuguang Hospital Affiliated to Shanghai University of Traditional Chinese Medicine, 528 ZhangHeng Road, Shanghai 201203, China

Full list of author information is available at the end of the article
}

collective term for any process causing the pathological changes to the structure of kidney which may consequently reduce renal function and disturbs the physiological homeostasis, a process collectively termed $[1,2]$. There is a continuous increase of the prevalence of CDK worldwide [3-5], which results in dramatic increasing incidences of end-stage renal disease (ESRD) [2] that require renal replacement therapy or undergoing dialysis due to lack of kidney donor.

Stem cell research and regenerative medicine provide a hope for treating CKD and even ERSD. However, it is extremely challenging to reconstruct human kidney because of its complicated anatomical structure and no regenerating zone of renal tissue to form new nephrons [6]. A variety insults could injure different renal cells including podocytes, tubular epithelial cells, mesangial cells, and endothelial cells. While the sublethal injuries 
damage renal function to different degrees, they could also activate the regenerating process for repairing the injuried kidney tissues [7]. It has been shown that renal dysfunction could be reversed by stimulating the angiogenic signaling and increasing renal microvasculature density $[8,9]$. Many animal studies have shown that renal cells could be modulated genetically or pharmacologically to promote kidney regeneration $[3,10]$.

Icariin, a flavonoid from plants of genus Epimedium, has been shown therapeutic potential for neurodegeneration, memory and depressive disorders, chronic inflammation, cardiovascular diseases, diabetes, osteoporosis, cancer, reproductive disorders, and immune dysfunction $[11,12]$. Icariin promoted self-renewal of mouse neural stem cells in vitro [13], the activation of quiescent neural stem cells of aged rats in vivo [14], and the differentiation of mesenchymal or stem cells into cardiomyocytes, osteoblasts, and endothelial cells [15-18]. The aim of this study was to investigate whether icariin could modulate renal stem cell population to repair kidney injury and alleviate chronic renal failure in a rat 5/6 nephrectomy model.

\section{Methods}

Animals

Eighty male Sprague Dawley rats (SPF class, $150 \pm 10$ g) were purchased from Shanghai Laboratory Animal Research Center (Shanghai, China) and kept in the laboratory animal center of Shanghai University of Traditional Chinese Medicine. The animals were given food and water ad libitho. All animal procedures were adhered to the Declaration of Helsinki and approved by the IACUC of Shanghai University of Traditional Chinese Medicine (SCXK (Shanghai) 2008-0016).

The renal failure rat model was established by a twophased procedure of 5/6 nephrectomy. Rats were given $3 \%$ sodium pentobarbital at $2 \mathrm{ml} / \mathrm{kg}$ dose intraperitoneally. After anesthesia, rats were put into left lateral position, shaved, disinfected with $75 \%$ ethanol. Locating the kidney under rib ridge and cutting a about $2 \mathrm{~cm}$ opening on skin, then cut the muscles; use tweezers to pull up the kidney out of surrounding fat, peel off renal cell membrane from the lower end (to avoid injury to adrenal gland); cut off about 5/6 renal tissue near both ends but keep parts around the renal hilum to avoid disruption of blood supply; wrap remaining kidney twice with a thick line and stretch $10 \mathrm{~min}$ with hemostat. When there was no further bleeding, the opening was sutured layer by layer and wiped clean with cotton swab. A week later the right renal hilum was ligated with thick lines to make kidney necrosis. The sham group rats were undergone the same procedure but without cutting any kidney tissue. The establishment of renal failure model was confirmed by serum creatinine level 2 weeks later.

\section{Treatments}

The rats were randomly put into sham, CKD model, and groups with 20 rats in each group. Sham and control rats were given water $3 \mathrm{ml} /$ day, icariin group were given icariin (40 mg/kg/d, CAS:489-32-7, Shanghai Winherb Medical Technology, Shanghai, China) in $3 \mathrm{ml} /$ day by gavage for 8 weeks. Then the rats were sacrificed by approved standard procedure, blood was collected via celiac artery, and kidney was divided into two sections longitudinally, one part for RNA work and the other for hematoxylin and eosin (H\&E) and immunohistochemical staining.

\section{Kidney function assays}

Serum urea nitrogen was measured by diacetyloxime colorimetric method, serum creatinine by picric acid method, and uric acid by colorimetric method.

\section{Kidney histology}

Kidney tissue was fixed in $10 \%$ formalin and sectioned followed by Hematoxylin and Eosin (H\&E) staining and light microscopy (Olympus IX70, Olympus, Shinjuku, Tokyo, Japan) observation.

\section{Reverse transcription and quantitative polymerase chain reaction (qPCR)}

Total mRNA was extracted from kidney tissue samples using Trizol (Life Technologies, Shanghai, China) method. The first strand cDNA was synthesized with $2 \mu \mathrm{g}$ total RNA using a reverse transcriptase kit from Biotnt (Shanghai, China) according to manufacturer's protocol. Quantitative PCR was performed in $20 \mu \mathrm{l}$ total reaction mix using Fast $\mathrm{SYBR}^{\circ}$ Green Master Mix (Life Technologies) on a ABI 7500 fast (Applied Biosystems, Foster City, CA) with $95{ }^{\circ} \mathrm{C}$ for 5 min followed by 40 cycles of $95{ }^{\circ} \mathrm{C} 5 \mathrm{~s}$ and $60{ }^{\circ} \mathrm{C} 30 \mathrm{~s}$. The sequences of specific primers were listed in Table 1 . The relative gene expression levels were calculated by $2^{-\Delta \Delta \mathrm{Ct}}$ method with $\beta$-actin as the internal control.

\section{Immunohistochemical (IHC) staining}

To detect the protein levels of TGF- $\beta 1$, HGF, and BMP7 and CD133 and CD24 positive cells in kidney tissue, IHC staining with specific antibodies was performed. The sections were deparaffinized and rehydrated, quenched with $3 \% \mathrm{H}_{2} \mathrm{O}_{2}$ for $10 \mathrm{~min}$, immersed in citrate buffer and heated in microwave at mid power for $3 \mathrm{~min}$, cooled down to room temperature and repeated heating another time before being washed twice in PBS at $5 \mathrm{~min}$ each and blocked with normal rabbit serum for $30 \mathrm{~min}$ at $37{ }^{\circ} \mathrm{C}$, followed by incubating with specified 1st antibodies at $4{ }^{\circ} \mathrm{C}$ over night and proper 2nd antibodies at $37{ }^{\circ} \mathrm{C}$ for $30 \mathrm{~min}$. The antibodies used were TGF- $\beta 1$, HGF, and BMP-7 from Abcam (Cambridge, 
Table 1 Sequences of qPCR primers used in this study

\begin{tabular}{|c|c|c|}
\hline Gene & Primer sequences & Amplicon (bp) \\
\hline \multirow[t]{2}{*}{ TGF- $\beta 1$} & GAAGGACCTGGGTTGGAAG & 136 \\
\hline & CGGGTTGTGTTGGTTGTAG & \\
\hline \multirow[t]{2}{*}{ HGF } & CCTATTTCCCGTTGTGAAG & 138 \\
\hline & ACTAACCATCCACCCTACT & \\
\hline \multirow[t]{2}{*}{ BMP-7 } & ACTACTGTGAGGGAGAGTG & 97 \\
\hline & TCTGGGTTGATGAAGTGAA & \\
\hline \multirow[t]{2}{*}{ Wt1 } & AAAAGTGGCTCACAGTGTC & 139 \\
\hline & ATGGAACAACCGCTCTAAT & \\
\hline \multirow[t]{2}{*}{ Pax-2 } & CTGGGCAGGTACTACGAGA & 190 \\
\hline & CGCTGGGAACTGTATCATT & \\
\hline \multirow[t]{2}{*}{ CD24 } & CCAGCCACCCCTGAGTAAATC & 90 \\
\hline & GAACTTAGTACCCGTGGTGAGTGA & \\
\hline \multirow[t]{2}{*}{ CD133 } & ACGGAAGTCAGCTCCCATCA & 76 \\
\hline & GGCTCTCCAGATCGGTTCTG & \\
\hline \multirow[t]{2}{*}{ Osr1 } & TTCTAAAGTGCCAGGTGCGG & 79 \\
\hline & GACGTGTGGAAACCAGGGAA & \\
\hline \multirow[t]{2}{*}{ Nanog } & TGCTCCGCTCCATAACTTCG & 100 \\
\hline & AGTGGCTTCCAAATTCGCCT & \\
\hline \multirow[t]{2}{*}{$\beta$-actin } & CTCACTGTCCACCTTCCAGC & 121 \\
\hline & AAGGGTGTAAAACGCAGCTCA & \\
\hline
\end{tabular}

MA), CD24 from Santa Cruz (Santa Cruz, CA), and CD133 from Boster (Wuhan, China). The secondary antibodies were purchased from Jackson ImmunoResearch Lab (West Grove, PA). The sections were washed 3 times with PBS before color development with a DAB kit (SA1020, Boster, Wuhan, China) and counterstained with hematoxylin, washed, dehydrated, cleared, and mounted. The slides were observed and photographed on a Olympus IX70 (Olympus, Shinjuku, Tokyo, Japan). The immunohistochemical staining results were semi quantitatively analyzed by combining the score of staining intensity and the score of percentage of positive cells. The positive staining ranged from pale yellow to brown. Staining intensity was scored as 0 for now color, 1 for pale yellow, 2 for yellow, 3 for brown. The percentage of positive cells was scored as 0 for 0 to $5 \%, 1$ for 6 to $25 \%, 2$ for 26 to $50 \%, 3$ for 51 to $75 \%$, and 4 for $>75 \%$. The final score was the product of the scores of staining intensity and the score of percentage of positive cells. Scores of 5 random fields (x400) were obtained from each slide.

\section{Statistical analyses}

Data was expressed as mean \pm standard deviation. Statistical analysis was performed using SPSS17.0 software package (IBM, Armonk, NY). The difference between the averages of groups was analyzed by one way ANOVA (analysis of variation), the homogeneity of variance between groups was determined by Levene's test and set 0.10 significance level; multiple comparisons between two groups were performed by SNK test if variances were assumed equal and by Tamhane's T2 test if variances were not assumed equal. A p value less than 0.05 was considered statistically significant.

\section{Results \\ Icariin (Icariin) alleviated the pathological changes of chronic kidney disease}

Hematoxylin and Eosin (H\&E) staining showed that kidney morphology was well preserved in sham operated rats (Fig. 1a). It showed normal glomerular structure, capillaries were not narrowed or occluded, capillary basement membrane lined well without enlargement; Bowman's capsule space was clear without expansion, glomerular capsule and glomerulus were separated; tubule structure was normal without protein cast or interstitial inflammatory cell infiltration.

Massive histological changes were observed in the kidneys of rats undergone 5/6 nephrectomy (model group) (Fig. 1b). The glomerular structure was disorganized with severe mesangial expansion, significant basement membrane thickening, and capillary compression or occlusion. Glomeruli had diffuse glomerular sclerosis with increased mesangial matrix area, reduced number of cells, glomerular fibrosis, and severe adhesions to Bowman's capsules. A
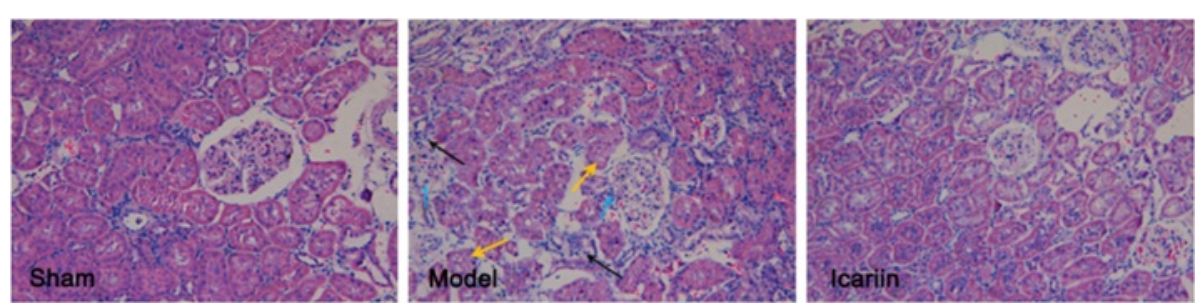

Fig. 1 Icariin alleviated chronic renal failure induced morphological damages to rat kidney. The kidney histology of rats underwent sham operation, 5/6 nephrectomy (CKD model), and CKD treated with icariin was observed by Hematoxylin and Eosin staining. The representative pictures were shown. Yellow arrows indicated tubular lesion and tubules lacking obvious brush border; black arrows indicated tubular necrosis and inflammatory cell infiltration; and green arrows indicated glomerular damages 
considerable amount of protein casts were observed in renal tubule lumen, which was significantly expanded with fibrosis and narrowing in some tubules. There was renal interstitial fibrosis and edema with large number of infiltrated inflammatory cells. Some small arteries showed intimal thickening, hyaline degeneration, and stenosis.

Compared to the model group, rats undergone 5/6 nephrectomy and received icariin treatment (Fig. 1c) had significantly reduced glomerular lesions with mild mesangial hyperplasia, mild basement membrane thickening, and unobvious glomerular sclerosis. Bowman's capsules had normal morphology without significant adhesions with glomeruli. The structure of renal tubules was nearly normal with mild renal tubular epithelial cell swelling, lumen expansion, mild stenosis without apparent protein casts, and few interstitial inflammatory cell infiltration.

\section{Icariin protected renal function against chronic kidney disease}

The blood urea nitrogen (BUN) level was increased from $6.084 \pm 0.574 \mathrm{mmol} / \mathrm{L}(95 \% \mathrm{CI}: 5.371-6.797 \mathrm{mmol} / \mathrm{L})$ in sham rats to $17.671 \pm 2.820 \mathrm{mmol} / \mathrm{L}$ (95\% CI: $15.653-$ $19.688 \mathrm{mmol} / \mathrm{L})$ in CKD model rats $(p<0.01)$. This increased level of BUN in CKD rats was significantly reduced by icariin (Fig. 2a). The levels of serum creatinine (Fig. 2b) and uric acid (Fig. 2c) had similar changes as BUN in CKD rats among different treatment groups.

Icariin promoted the expression of genes related to stem cell proliferation

Chronic kidney disease and icariin treatment each triggered changes of the expression of genes involved in stem cell/renal progenitor cell proliferation (Figs. 3 and 4). The renal mRNA levels of TGF- $\beta 1$ (Fig. 3a), HGF (Fig. 3b), and WT-1 (Fig. 3d) were significantly higher whereas renal mRNA levels of BMP-7 (Fig. 3c) and Pax-2 (Fig. 3e) were significantly lower in CKD rats compared with those of healthy rats. Icariin drastically reduced the mRNA level of TGF- $\beta 1$ (Fig. 3a) while increased the levels of HGF
(Fig. 3b), BMP-7 (Fig. 3c), WT-1 (Fig. 3d), and Pax-2 (Fig. 3e) in CKD rats.

Consistently, the protein level of TGF- $\beta 1$ was strongly elevated by injury-induced CKD, which was markedly inhibited by icariin and to a lesser extent by losartan (Fig. 4a). The HGF protein level in the residual kidney of CKD rats was significantly increased by icariin (Fig. 4b) while BMP-7 protein level in CKD rats was only recovered back to about the normal rats by icariin from more than 50 \% decrease in CKD rats (Fig. 4c).

\section{Icariin promoted the proliferation of renal stem/ progenitor cells}

The mRNA levels of markers of renal stem cell CD133 (Fig. 5a), CD24 (Fig. 5b), and Osr1 (Fig. 5c) and pluropotency (Nanog, Fig. 5d) were increased in the remnant kidney of 5/6 nephrectomy CKD rats and further increased 3-5 folds by icariin treatment (Fig. 5).

The number of $\mathrm{CD} 133^{+}$renal cells decreased about $65 \%$ in CKD rats compared to healthy rats and icariin treatment resulted in about 5 fold increase of $\mathrm{CD}_{133^{+}}$ cells in the kidney of CKD rats (Fig. 6a). The numbers of CD24+ cells had similar changes among healthy rats, CKD rats, and icariin or losartan- treated rats (Fig. 6b). Meanwhile, the numbers of renal stem/progenitor cells were correlated with kidney functions (Table 2), especially, $\mathrm{CD}_{133^{+}}$cell number showed tight negative correlation with BUN and creatinine levels.

\section{Discussions}

Injury-induced chronic kidney disease caused profound histological changes including glomeruli damages, tubular necrosis, and infiltration of inflammatory cells in rat kidneys, which was accompanied with loss of renal functions indicated by the increase of BUN, creatinine, and uric acid levels. Icariin alleviated all such pathological changes, e. g. seldom tubular damages and much less immune cell infiltration, complete glomeruli structure, and nearly normal levels of BUN, creatinine, and uric acid in CKD rats received icariin. Furthermore, icariin

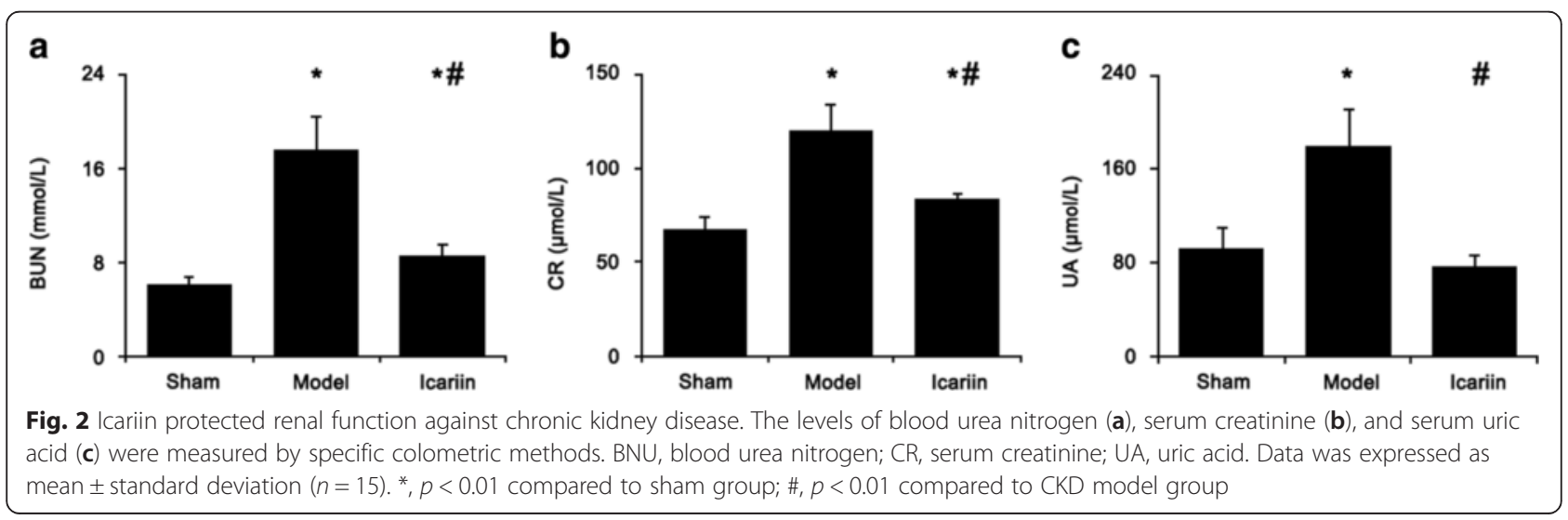



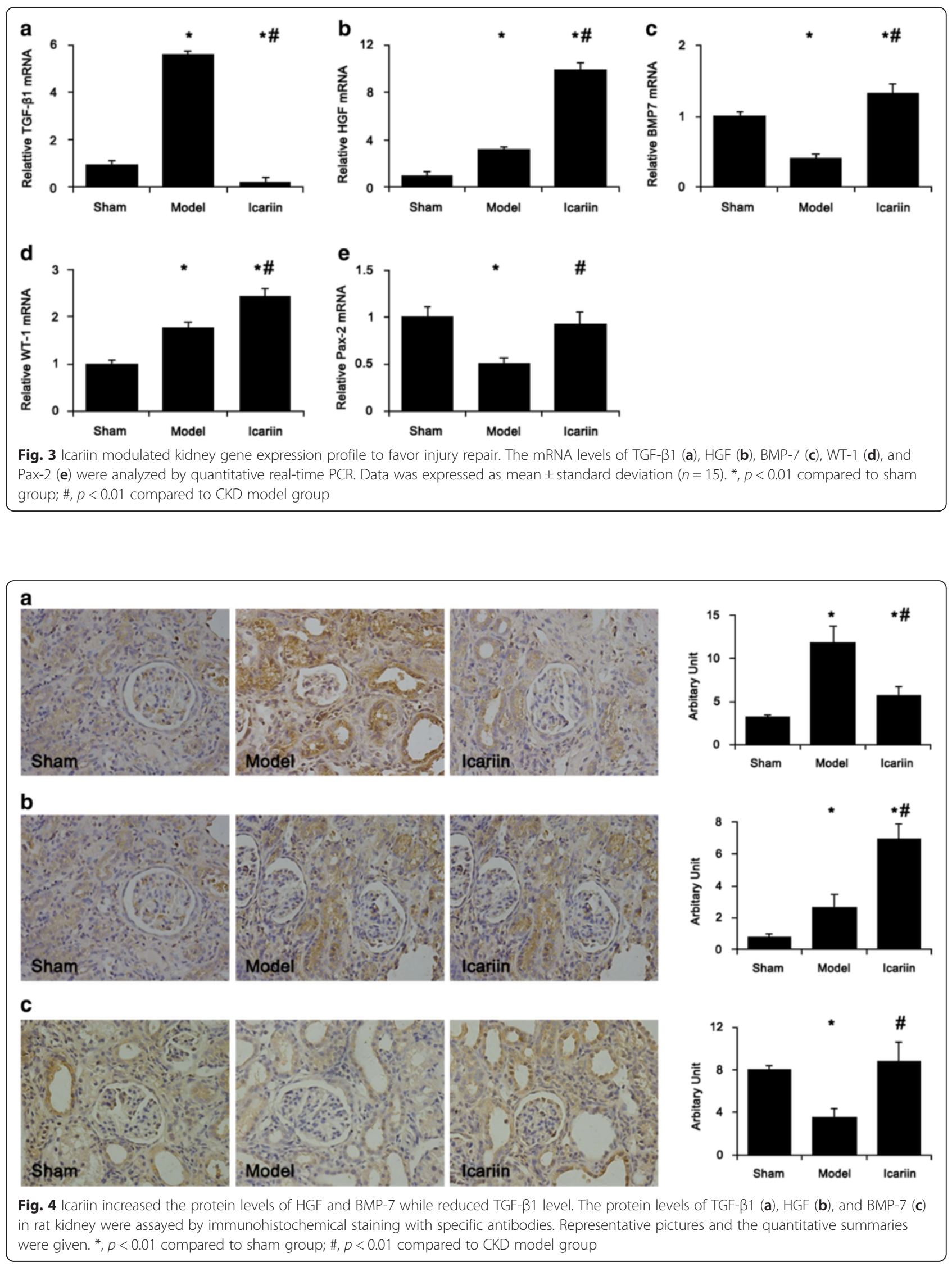

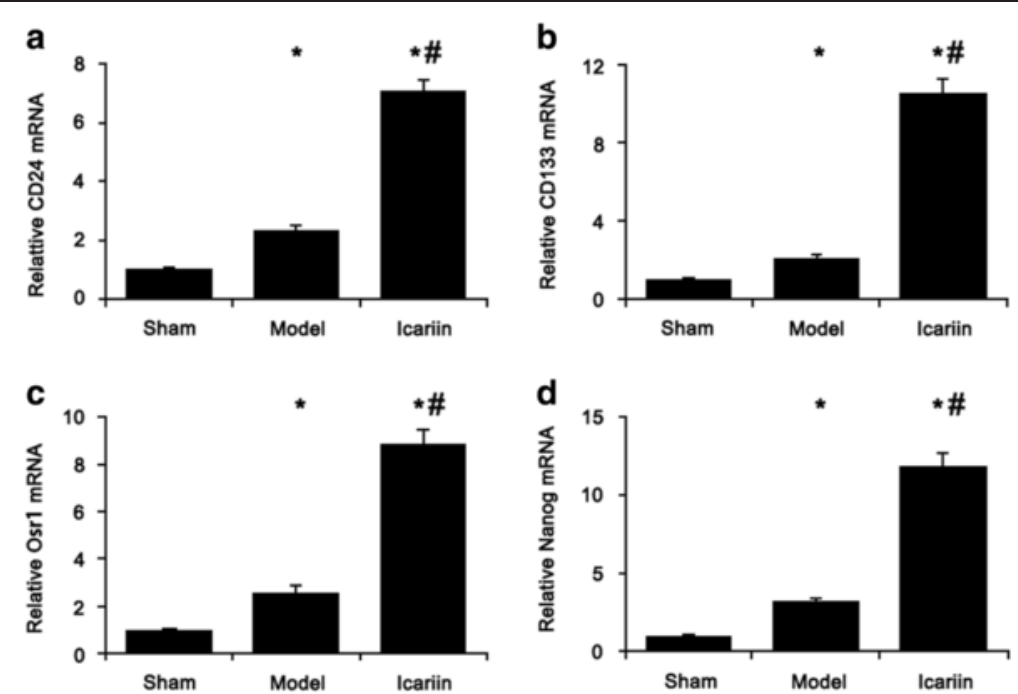

Fig. 5 Icariin up-regulated the expression of renal stem cell markers. The mRNA levels of CD133 (a), CD24 (b), Osr1 (c), and Nanog (d) in the remnant kidney were analyzed by quantitative real-time PCR. Data was expressed as mean \pm standard deviation $(n=15)$. ${ }^{*}, p<0.01$ compared to sham group; \#, $p<0.01$ compared to CKD model group

significantly increased the numbers of $\mathrm{CD}_{133^{+}}$and $\mathrm{CD}_{2} 4^{+}$renal stem/progenitor cells in injury-induced CKD rats. Icariin activated the expression of genes promoting renal stem/progenitor cells proliferation and inhibited the expression of fibrosis-promoter TGF- $\beta 1$.

Renal regeneration is at the center of treating chronic kidney failure and other kidney diseases $[10,19,20]$. As icariin was renal protective by changing cell cycle distribution of renal cells [21] and was shown to promote the maintenance, activation, and differentiation of many types of stem cells [13-18], we postulated that the action of icariin in improving renal function would involve the activation of pathways promoting the proliferation and/ or homing of renal stem/progenitor cells (Fig. 7). Among the factors involved in kidney development and kidney injury recovery, hepatocyte growth factor (HGF) and bone morphogenetic protein-7 (BMP-7) were analyzed and both found significantly increased by icariin in CKD rat kidney tissues. HGF/HGF receptor c-met signaling conferred renal protective effect by preventing apoptosis, reducing inflammation, and inhibiting TGF- $\beta$-induced fibrosis $[22,23]$. BMP-7 promoted kidney regeneration

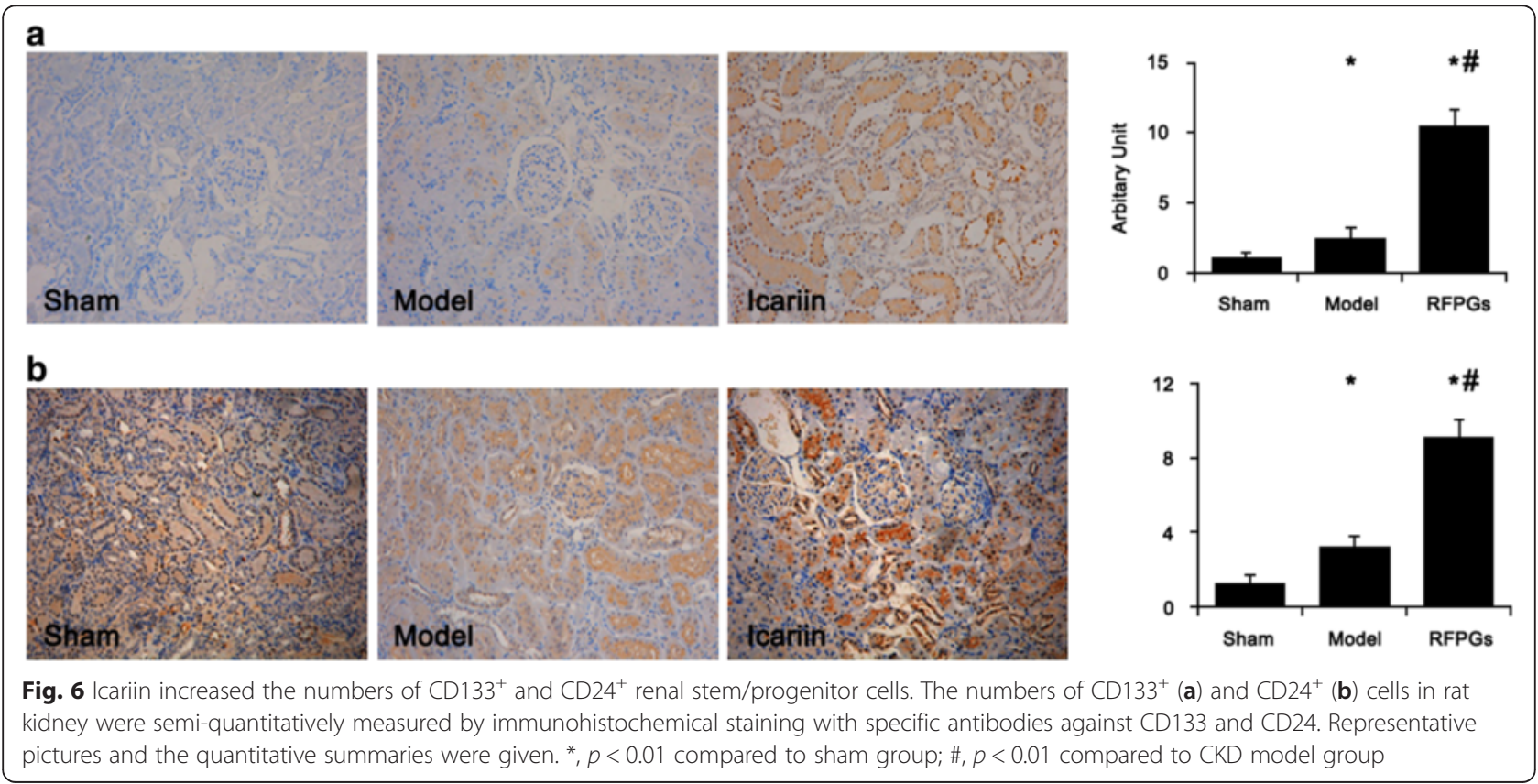


Table 2 Correlation between renal function and the number of renal stem/progenitor cells

\begin{tabular}{llllll}
\hline & & BUN & Cr & CD133 & CD24 \\
\hline BUN & Pearson Correlation & 1 & $0.966^{* *}$ & $-0.979^{* *}$ & $-0.912^{*}$ \\
& Sig. (1-tailed) & & 0.004 & 0.002 & 0.015 \\
$\mathrm{Cr}$ & Pearson Correlation & $0.966^{* *}$ & 1 & $-0.946^{* *}$ & -0.799 \\
& Sig. (1-tailed) & 0.004 & & 0.008 & 0.053 \\
CD133 & Pearson Correlation & $-0.979^{* *}$ & $-0.946^{* *}$ & 1 & $0.935^{* *}$ \\
& Sig. (1-tailed) & 0.002 & 0.008 & & 0.01 \\
CD24 & Pearson Correlation & $-0.912^{*}$ & -0.799 & $0.935^{* *}$ & 1 \\
& Sig. (1-tailed) & 0.015 & 0.053 & 0.01 & \\
\hline
\end{tabular}

BUN blood urea nitrogen; $\mathrm{Cr}$ serum creatinine

${ }^{*}, p<0.05 ;{ }^{* *}, p<0.01$

by inhibiting the precocious differentiation of the kidney progenitor cells and antagonizing the TGF- $\beta$ in inducing fibrosis [24, 25]. Moreover, icariin significantly upregulated the expression of genes important for the proliferation and differentiation of renal stem/progenitor cells (Osr1, NMP-7, Pax2 and WT-1), which led to the increase of renal $\mathrm{CD}_{133^{+}}$and/or $\mathrm{CD} 24^{+}$stem/progenitor cells. Renal $\mathrm{CD}_{13}{ }^{+} \mathrm{CD} 24^{+} \mathrm{PDX}-$ cells were shown to be the uncommitted stem cells which possessed the potentials of self renewal and differentiation into both podocytes and tubular cells $[26,27]$. The elevation of Osr1, Nanog, HGF, BMP-7, WT-1, and Pax2 expression in CKD rat kidney by Icariin was well in correlation with the increased numbers of $\mathrm{CD}_{133^{+}}$and/or $\mathrm{CD} 24^{+}$stem/progenitor cells while the reduced expression of TGF- $\beta 1$ leading to the blocking of interstitial fibrosis (Fig. 7).

\section{Conclusions}

In summary, icariin attenuated the progression of subtotal nephrectomy induced chronic renal failure in rats. Icariin moderated the loss of the integrity of kidney histology and renal function in CKD rats. Those structural and functional benefits of icariin were accompanied by the increased expression of genes promoting the proliferation of renal stem/progenitor cells as well as the increase

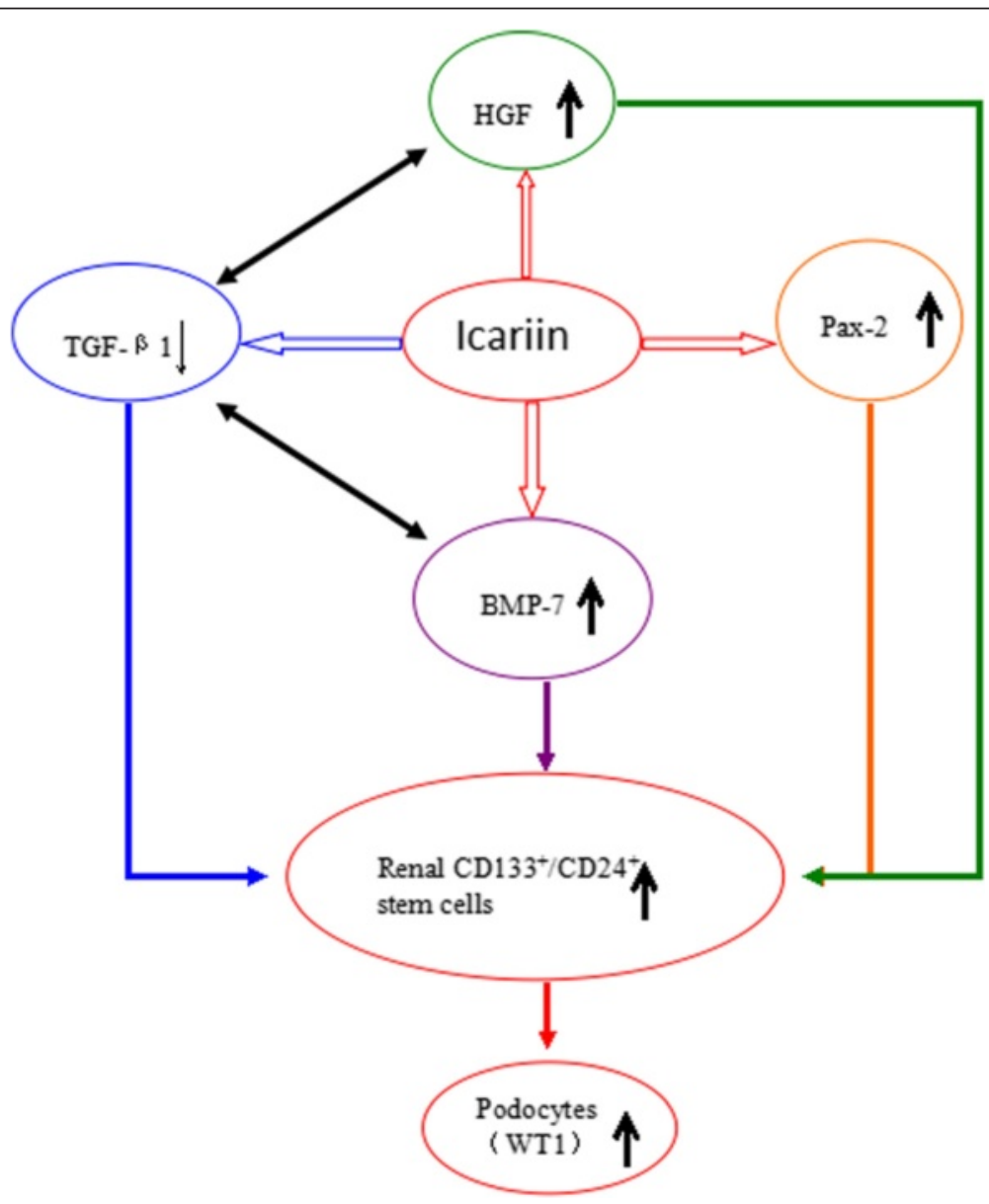

Fig. 7 Proposed model for the actions of icariin in alleviating CKD. Icariin activates the expression of genes promoting the proliferation, survival, and differentiation of renal stem cells (e. g. HGF, BMP-7, Pax-2, and WT-1) and inhibits genes promoting inflammation and fibrosis (e. g. TGF- $\beta 1$ ), which leads to the increase of renal stem/progenitor cells and the repair of kidney injuries 
of the numbers of $\mathrm{CD}_{133^{+}}$and/or $\mathrm{CD} 24^{+}$renal stem/progenitor cells.

\section{Abbreviations}

CKD: Chronic kidney disease; ESRD: End-stage renal disease;

H\&E: Hematoxylin and Eosin; qPCR: Quantitative polymerase chain reaction; IHC: Immunohistochemistry; BUN: Blood urea nitrogen.

\section{Competing interests}

The authors declare no conflicts of interest.

\section{Authors' contributions}

$\mathrm{ZH}$ carried out experiments, analyzed data, and drafted the manuscript. $\mathrm{JH}$ and DH carried out animal experiments. SL and JG conceived the project, designed study, drafted the manuscript and revised it. All authors have read and approved the final manuscript.

\section{Author details}

'Department of Hematology, Shuguang Hospital Affiliated to Shanghai University of Traditional Chinese Medicine, 185 Pu'an Road, Shanghai 200021, China. ${ }^{2}$ Department of nephrology, Shuguang Hospital Affiliated to Shanghai University of Traditional Chinese Medicine, 528 ZhangHeng Road, Shanghai 201203, China. ${ }^{3}$ Traditional Chinese Medicine Institute of Kidney Diseases, Shanghai University of Traditional Chinese Medicine, 528 ZhangHeng Road, Shanghai 201203, China. ${ }^{4}$ Shanghai Key Laboratory of Traditional Chinese Clinical Medicine, 528 ZhangHeng Road, Shanghai 201203, China. ${ }^{5}$ Collage of Biology and pharmacy, China Three Gorges University, 8 Daxue Road, Yichang, Hubei 443002, China.

Received: 22 July 2015 Accepted: 11 October 2015

Published online: 21 October 2015

\section{References}

1. Dziedzic K, Pleniceanu O, Dekel B. Kidney stem cells in development, regeneration and cancer. Semin Cell Dev Biol. 2014;36:57-65.

2. Faa G, Sanna A, Gerosa C, Fanni D, Puddu M, Ottonello G, et al. Renal physiological regenerative medicine to prevent chronic renal failure: Should we start at birth? Clin Chim Acta. 2015;444:156-62.

3. Chou YH, Pan SY, Yang CH, Lin SL. Stem cells and kidney regeneration. J Formos Med Assoc. 2014;113:201-9.

4. Coresh J, Selvin E, Stevens LA, Manzi J, Kusek JW, Eggers P, et al. Prevalence of chronic kidney disease in the United States. JAMA. 2007;298:2038-47.

5. Stauffer ME, Fan T. Prevalence of anemia in chronic kidney disease in the United States. PLoS One. 2014;9:e84943.

6. Hopkins C, Li J, Rae F, Little MH. Stem cell options for kidney disease. J Pathol. 2009;217:265e81.

7. Humphreys BD, Valerius MT, Kobayashi A, Mugford JW, Soeung S, Duffield JS, et al. Intrinsic epithelial cells repair the kidney after injury. Cell Stem Cell. 2008;2:284e91.

8. Chade AR, Kelsen S. Reversal of renal dysfunction by targeted administration of VEGF into the stenotic kidney: a novel potential therapeutic approach. Am J Physiol Renal Physiol. 2012;302:F1342-1350.

9. Long DA, Norman JT, Fine LG. Restoring the renal microvasculature to treat chronic kidney disease. Nat Rev Nephrol. 2012;8:244-50.

10. Benigni A, Morigi M, Remuzzi G. Kidney regeneration. Lancet. 2010;375:1310-7.

11. Schluesener JK, Schluesener H. Plant polyphenols in the treatment of ageassociated diseases: revealing the pleiotropic effects of icariin by network analysis. Mol Nutr Food Res. 2014;58:49-60.

12. Li C, Li Q, Mei Q, Lu T. Pharmacological effects and pharmacokinetic properties of icariin, the major bioactive component in Herba Epimedii. Life Sci. 2015:126:57-68.

13. Huang JH, Cai WJ, Zhang XM, Shen ZY. Icariin promotes self-renewal of neural stem cells: an involvement of extracellular regulated kinase signaling pathway. Chin J Integr Med. 2014;20:107-15.

14. Wu B, Chen Y, Huang J, Ning Y, Bian Q, Shan Y, et al. Icariin improves cognitive deficits and activates quiescent neural stem cells in aging rats. J Ethnopharmacol. 2012;142:746-53.

15. Zhou L, Huang Y, Zhang Y, Zhao Q, Zheng B, Lou Y, et al. mGluR5 stimulating Homer-PIKE formation initiates icariin induced cardiomyogenesis of mouse embryonic stem cells by activating reactive oxygen species. Exp Cell Res. 2013;319:1505-14.
16. Zhai $Y K$, Guo $X Y$, Ge BF, Zhen $P, M a X N$, Zhou J, et al. Icariin stimulates the osteogenic differentiation of rat bone marrow stromal cells via activating the PI3K-AKT-eNOS-NO-cGMP-PKG. Bone. 2014;66:189-98.

17. Wu $Y, X i a ~ L, Z h o u ~ Y, X u Y$, Jiang $X$. Icariin induces osteogenic differentiation of bone mesenchymal stem cells in a MAPK-dependent manner. Cell Prolif. 2015;48:375-84

18. Tang $Y$, Jacobi A, Vater C, Zou L, Zou X, Stiehler M. Icariin promotes angiogenic differentiation and prevents oxidative stress-induced autophagy in endothelial progenitor cells. Stem Cells. 2015;33:1863-77.

19. Maeshima A, Nakasatomi M, Nojima Y. Regenerative medicine for the kidney: renotropic factors, renal stem/progenitor cells, and stem cell therapy. Biomed Res Int. 2014;2014:595493.

20. Morigi M, Benigni A. Mesenchymal stem cells and kidney repair. Nephrol Dial Transplant. 2013;28:788-93

21. Liang SR, Bi JW, Guo ZL, Bai Y, Hu Z. Protective effect of icariin on kidney in 5/6 nephrectomized rats and its mechanism. Genet Mol Res. 2014;13:6466-71.

22. Yazawa K, Isaka Y, Takahara S, Imai E, Ichimaru N, Shi Y, et al. Direct transfer of hepatocyte growth factor gene into kidney suppresses cyclosporin A nephrotoxicity in rats. Nephrol Dial Transplant. 2014;19:812-6.

23. Zhou D, Tan RJ, Lin L, Zhou L, Liu Y. Activation of hepatocyte growth factor receptor, c-met, in renal tubules is required for renoprotection after acute kidney injury. Kidney Int. 2013;84:509-20.

24. Tomita M, Asada M, Asada N, Nakamura J, Oguchi A, Higashi AY, et al. Bmp7 maintains undifferentiated kidney progenitor population and determines nephron numbers at birth. PLoS One. 2013;8:e73554.

25. Lv S, Liu G, Sun A, Wang J, Cheng J, Wang W, et al. Mesenchymal stem cells ameliorate diabetic glomerular fibrosis in vivo and in vitro by inhibiting TGF- $\beta$ signalling via secretion of bone morphogenetic protein 7. Diab Vasc Dis Res. 2014;11:251-61.

26. Ronconi E, Sagrinati C, Angelotti ML, Lazzeri E, Mazzinghi B, Ballerini L, et al. Regeneration of glomerular podocytes by human renal progenitors. J Am Soc Nephrol. 2009;20:322-32.

27. Romagnani P, Remuzzi G. CD133+ renal stem cells always co-express CD24 in adult human kidney tissue. Stem Cell Res. 2014;12:828-9.

\section{Submit your next manuscript to BioMed Central and take full advantage of:}

- Convenient online submission

- Thorough peer review

- No space constraints or color figure charges

- Immediate publication on acceptance

- Inclusion in PubMed, CAS, Scopus and Google Scholar

- Research which is freely available for redistribution 